Check for updates

Cite this: Analyst, 2021, 146, 529

\title{
Rapid detection of mozzarella and feta cheese adulteration with cow milk through a silicon photonic immunosensor $\uparrow$
}

\author{
Michailia Angelopoulou, ${ }^{a}$ Panagiota S. Petrou, ${ }^{a}$ Ioannis Raptis, ${ }^{b}$ \\ Konstantinos Misiakos, ${ }^{b}$ Evangelia Livaniou, ${ }^{c}$ Eleni Makarona*b and \\ Sotirios Kakabakos iD *a
}

\begin{abstract}
Mozzarella di Bufala Campana and Feta are two cheeses with Protected Designation of Origin the fraudulent adulteration of which with bovine milk must be routinely checked to ensure that consumers actually buy these high-end products and avoid health issues related to bovine milk allergy. Here, we employed, for the first time, a silicon-based photonic immunosensor for the detection of mozzarella and feta adulteration with bovine milk. The photonic immunosensor used relies on Mach-Zehnder interferometers monolithically integrated along with their respective light sources on a silicon chip. A rabbit polyclonal antiserum raised against bovine $\kappa$-casein was used for the development of a competitive immunoassay realized in three steps, including a reaction with the antiserum, a biotinylated anti-rabbit IgG antibody, and streptavidin. The implementation of this assay configuration significantly reduced the non-specific signal due to the cheese matrix, and allowed completion of the assay in $\sim 9$ min. After optimization of all assay conditions, bovine cheese could be quantified in mozzarella or feta at concentrations as low as 0.5 and $0.25 \%(\mathrm{w} / \mathrm{w})$, respectively; both quantification limits were below the maximum allowable content of bovine milk in mozzarella and feta $(1 \% \mathrm{w} / \mathrm{w})$ according to the EU regulations. Equally important, the assays were reproducible with intra- and inter-assay coefficients of variation $<10 \%$, and exhibited a wide linear dynamic range that extended up to 50 and $25 \%$ (w/w) for mozzarella and feta, respectively. Taking into account its performance, the proposed immunosensor may be transformed to a new tool against fraudulent activities in the dairy industry.
\end{abstract}

Received 25th August 2020 Accepted 15th October 2020

DOI: 10.1039/d0an01706j

rsc.li/analyst day diet or are considered high-end products to be exported. The origin and composition of milk affects the organoleptic properties of the cheese, ${ }^{1}$ while the higher price and seasonal availability of milk obtained from species other than cattle are the reasons of incidents of fraudulent addition of cow milk during cheese manufacturing to reduce production cost. ${ }^{2-4}$ Identification of cheese authenticity is mandatory for the protection of consumers who avoid the consumption of cow milk due to allergy or religious/cultural reasons. ${ }^{5,6}$ In parallel, high-quality cheeses produced exclusively from milk of other species than cattle have been registered according to the law as Protected Designation of Origin (PDO) products. For this reason, the EU and US legislations require the food industry to declare the species origin of milk used for cheese preparation. ${ }^{7,8}$ In particular, the maximum acceptable content of cow milk in cheese from other species set by the European commission (EC) is $1 \%(\mathrm{w} / \mathrm{w}){ }^{9}$

According to the EC, the reference method for goat, sheep and buffalo dairy product adulteration with cow milk is performed through isoelectric focusing of $\gamma$-caseins. ${ }^{10}$ To protect consumers from misleading inscription of ingredients labelled 
on the product package, several methods such as electrophoresis, chromatography, mass spectrometry, liquid chromatography-mass spectrometry and matrix-assisted laser desorption/ionization time-of-flight mass spectrometry (MALDI-ToFMS) have been developed for the detection of milk or cheese fraud. ${ }^{11-18}$ In recent years, single or multiplex PCR techniques have also been reported for authenticity confirmation of cheeses made from the milk of goat, sheep, buffalo, or a mixture of them. ${ }^{4,19-21}$ Nevertheless, all of the above methods are time consuming and labor intensive and require expensive instrumentation. On the other hand, enzyme-linked immunosorbent assays (ELISA) employing either monoclonal or polyclonal antibodies against bovine caseins, whey proteins, bovine immunoglobulins and caseinomacropeptides are used for the detection of dairy product adulteration providing the potential for multiple samples' analysis in a single run. ${ }^{22-24}$ In addition, immunochromatographic methods, such as lateral flow devices and dipstick tests, have been developed and commercialized as tools for on-site analysis providing, however, semi-quantitative results. ${ }^{24-26}$

Immunosensors have emerged as alternative methods for adulteration detection in order to combine the quantitative determination offered by ELISA with the on-site detection capability of immunochromatographic methods. Depending on the transducer used, biosensors are distinguished as electrochemical, piezoelectric and optical ones. ${ }^{27-31}$ The latter offer several competitive advantages, such as signals that are less vulnerable to interference, since the detector is isolated from the sample, the potential of miniaturization and as a corollary the system compactness and portability as well as multianalyte detection schemes. Even more specifically, label-free immunosensors are suitable for real-time and on-site analysis, since they provide direct signal transduction and highthroughput screening. ${ }^{32}$ Interferometric sensors in their various formats, e.g., Mach-Zehnder, Young or Bimodal interferometers, single beam and other grating coupled interferometers, are amongst the most sensitive sensing approaches reported in the literature. ${ }^{33-35}$ Immunosensors based on surface plasmon resonance (SPR) and Mach-Zehnder Interferometry (MZI) have already been used for detection of goat/sheep milk adulteration with bovine milk. ${ }^{36,37}$ Nonetheless, to the best of our knowledge, there are no reports in the literature regarding the detection of cheese adulteration with cow milk by employing immunosensors.

In this work, we present for the first time a label-free optical immunosensor for the rapid and accurate determination of adulteration of two PDO cheeses with bovine milk, namely Mozzarella di Bufala Campana and Greek feta, exploiting Broad-Band MZIs (BB-MZIs) monolithically integrated onto silicon chips. Each chip accommodates 10 planar silicon nitride ridge waveguides along with their respective broadband (BB) silicon avalanche diodes (LEDs). The BB-MZIs are covered by a silicon oxide cladding layer that has been removed from a $600 \mu \mathrm{m}$-long area over the sensing arm to allow for interaction of the waveguided modes with the sample. The 10 BB-MZIs of the chip converge to a single point a

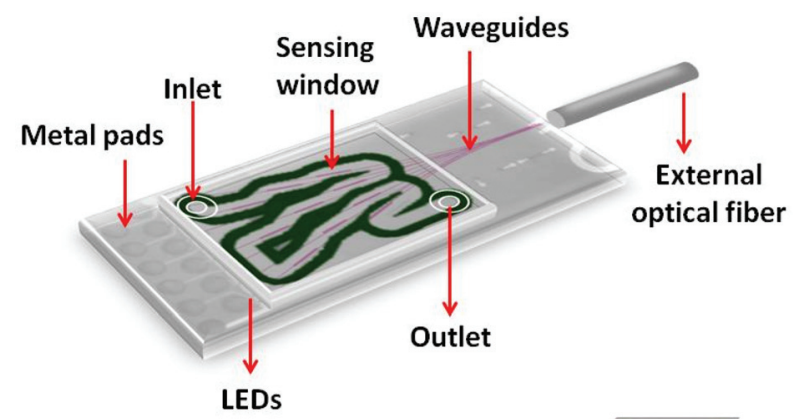

b

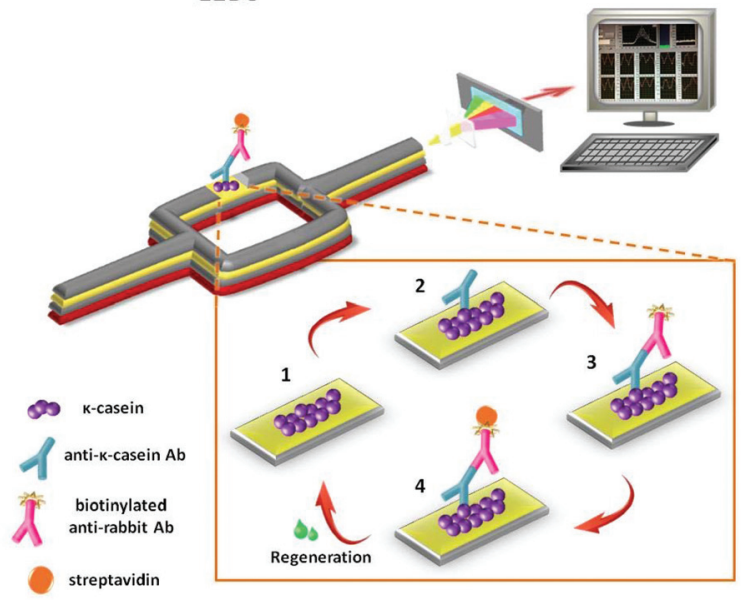

Fig. 1 (a) Schematic of the chip with the fluidic on top and the positioning of the external optical fiber used for the collection of the transmitted spectra of the integrated on chip MZIs. (b) Schematic of a single $\mathrm{MZI}$ and of the immunoassay steps for detection of bovine $\mathrm{\kappa}$-casein in buffalo mozzarella and feta cheese.

where an external optical fiber is positioned to collect the transmitted interference spectrum and guide it to a spectrometer (Fig. 1a). The transmission spectra of the 10 BB-MZIs are serially recorded within $10 \mathrm{~s}$, a function that is controlled by an electrical multiplexer. Biomolecular reactions taking place on the sensing arm change the effective refractive index on the waveguide surface resulting in a blue shift of the interference spectrum, thus, providing a way to monitor the biomolecular adlayer growth on the sensing arm. BB-MZIs have been exploited for several applications including goat milk adulteration with bovine milk, ${ }^{37}$ determination of C-reactive protein in human serum, ${ }^{38}$ mycotoxins in beer, ${ }^{39}$ and allergens in food industry rinsing water. ${ }^{40}$ To achieve the goal of the present work, a polyclonal antibody against bovine $\kappa$-casein was raised in rabbits and employed for the development of immunoassay. Due to the polyclonal nature of the developed antibody it was possible to detect the casein fragments, i.e., the caseinomacropeptide which is a whey-peptide produced during cheese making. ${ }^{41}$ The antiserum produced was characterized in terms of titer and specificity against all milk proteins. On-chip detection of cheese adulteration was achieved by biofunctionalizing the sensing arm of MZI with bovine $\kappa$-casein and following a competitive immunoassay format (Fig. 1b). All assay parameters were optimized, while 
special emphasis was given to the assay configuration so as to alleviate the matrix effects without compromising the assay duration and analytical performance. The assay was quite fast and completed within $9 \mathrm{~min}$ with a dynamic range that enabled quantitative detection of bovine milk in mozzarella and feta cheeses at concentrations lower than the maximum allowable level $(1 \% \mathrm{w} / \mathrm{w})$ set by the EC regulations for PDO cheeses.

\section{Materials and methods}

\subsection{Reagents}

Commercial feta cheese (DELTA S.A.), buffalo mozzarella (Mozzarella di Bufala Campana, Fattorie Garofalo) and the respective cheeses made of cow milk (white cheese Polykastrou, KOLIOS S.A.; mozzarella Dirollo, OPTIMA S.A.) were purchased from the local market. Bovine $\kappa^{-}, \beta-$, and $\alpha$-casein, $\alpha$-lactalbumin, $\beta$-lactoglobulin from bovine milk, bovine serum albumin (BSA), bovine immunoglobulins, goat anti-rabbit IgG antibody, streptavidin, and 3-aminopropyltriethoxysilane (APTES) were purchased from Sigma-Aldrich (Darmstadt, Germany). All other reagents were of analytical grade and were purchased from Sigma-Aldrich. The Bio-Shield Cow Cheese ELISA kit from ProGnosis Biotech S.A. (Larissa, Greece) was used as a comparison method for the detection of adulteration of commercially available buffalo mozzarella and feta cheeses with bovine milk. The water used for the study was doubly-distilled. Biotinylation of goat anti-rabbit IgG antibody was performed following a previously published protocol. $^{42}$

\subsection{Anti-bovine $\kappa$-casein rabbit antiserum production and characterization}

The antiserum was developed in-house following a previously described immunization procedure, properly modified. ${ }^{43}$ Briefly, two New Zealand white rabbits were immunized with bovine- $\kappa$-casein (100 $\mu \mathrm{g}$ per animal) emulsified with Freund's adjuvant and administered through multiple intradermal injections. The first immunization was followed by four booster ones, performed four, seven, ten and thirteen weeks after initial exposure. Blood was collected from the central ear vein 10-14 days after each booster immunization. Antiserum was obtained by centrifugation of whole blood. The immunization protocol was approved by the Greek Authorities and the local committee of the Animal House (Institute of Biosciences \& Applications, NCSR "Demokritos"), where the experimental procedure was performed. Animal experimentation was performed in accordance with the Presidential Decree 56/2013 for the Protection of Animals used for Scientific Purposes (Directive 2010/63/EU) and approved by the local committee of the Animal House (Institute of Biosciences \& Applications, NCSR "Demokritos") which is a certified installation (EL 25 BIOexp 039, Prefecture of Attica) and the Prefecture of Attica, Division of Agriculture and Veterinary Medicine (license No 1717/24-03-2017).

\subsection{Preparation of cheese calibrators}

Buffalo mozzarella and feta cheese were tested for adulteration with bovine milk using a commercially available ELISA kit with a LOD of $0.04 \%$. It was found that the PDO products used did not contain bovine milk at detectable concentrations, and thus they were used as matrices for the preparation of calibrators. Calibrators for mozzarella were prepared by mixing certain amounts of cow mozzarella to buffalo mozzarella so as to achieve concentrations ranging from 0.5 to $50 \%(\mathrm{w} / \mathrm{w})$; feta calibrators were prepared by mixing white cheese (prepared from cow milk in a manner similar to feta) with feta to obtain concentrations of 0.25 to $25 \%(\mathrm{w} / \mathrm{w})$. The mixtures were homogenized using a pestle and mortar, and $1 \mathrm{~g}$ from the homogenate was mixed with $1 \mathrm{~mL}$ of $10 \mathrm{mM}$ PBS, pH 7.4. After centrifugation at $2000 \mathrm{~g}$ for $10 \mathrm{~min}$, the supernatant was collected and diluted 50 times with $10 \mathrm{mM}$ PBS, $\mathrm{pH}$ 7.4, containing $0.5 \%(\mathrm{w} / \mathrm{v}) \mathrm{BSA}$ and $0.9 \%(\mathrm{w} / \mathrm{v}) \mathrm{NaCl}$ (assay buffer).

\subsection{Biofunctionalization of the chips}

Silicon chips $\left(8.0 \times 4.2 \mathrm{~mm}^{2}\right)$ containing arrays of 10 BB-MZIs along with the respective light sources have been manufactured as described in previous publications. ${ }^{37-40}$ The protocol for chip biofunctionalization has been described previously. ${ }^{37}$ Briefly, the cleaning and hydrophylization of silicon chips was performed by $\mathrm{O}_{2}$ plasma treatment $(10 \mathrm{mTorr})$ for $30 \mathrm{~s}$ followed by aminosilanization through immersion for $2 \mathrm{~min}$ in a $0.5 \%(\mathrm{v} / \mathrm{v})$ APTES solution. Then, the chips were washed with water, dried under a nitrogen stream, and heated at $120^{\circ} \mathrm{C}$ for $20 \mathrm{~min}$. The biological modification of the chip was performed using the BioOdyssey Calligrapher Mini Arrayer (Bio-Rad Laboratories, Inc.). Seven MZIs on each chip were spotted with $100 \mu \mathrm{g} \mathrm{mL}{ }^{-1} \kappa$-casein solution in coating buffer, whereas the

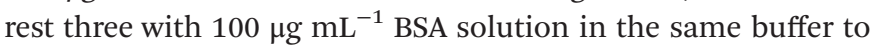
allow determination of the non-specific binding signal. After the completion of spotting, the chips were left in a controlled humidity chamber (75\% humidity) overnight at $4{ }^{\circ} \mathrm{C}$. Then, the chips were washed with $10 \mathrm{mM}$ phosphate buffer, $\mathrm{pH} 7.4$ (washing buffer), blocked with $10 \mathrm{~g} \mathrm{~L}^{-1} \mathrm{BSA}$ in $0.1 \mathrm{M} \mathrm{NaHCO}_{3}$, pH 8.5, for 1 hour, washed again, and dried under a nitrogen stream and were ready for use.

\subsection{Assay for the detection of bovine cheese in feta and buffalo mozzarella with the MZI immunosensor}

The delivery of the reagents over the modified chip surface was performed by attaching on the chip microfluidic modules that have been selected according to a suitable fluidic chamber geometry (Fig. S1a $\dagger$ ). ${ }^{40}$ The microfluidic-covered chip was then placed in the docking station of the measuring apparatus (Fig. S1b $\dagger$ ). An injector (Rheodyne 7725i) equipped with a $100 \mu \mathrm{L}$ loop was used for the introduction of reagents in flow. For the assay, the 50-times diluted cheese calibrators were mixed with a $1: 150$ dilution of rabbit anti- $\kappa$-casein antiserum at $1: 1$ volume ratio and incubated for $30 \mathrm{~min}$. Then, $100 \mu \mathrm{L}$ of each calibrator/antiserum mixture were passed over the chip, followed by $100 \mu \mathrm{L}$ of a $10 \mu \mathrm{g} \mathrm{mL}^{-1}$ biotinylated anti-rabbit IgG 
antibody solution and $100 \mu \mathrm{L}$ of $10 \mu \mathrm{g} \mathrm{mL}^{-1}$ streptavidin solution in assay buffer using a peristaltic pump. The flow rate was $33 \mu \mathrm{L} \mathrm{min}{ }^{-1}$ resulting in a total assay time of approximately $9 \mathrm{~min}$. After the completion of the assay a regeneration step was performed by passing sequentially over the chip $100 \mu \mathrm{L}$ of $40 \mathrm{mM} \mathrm{NaOH}$ and $100 \mu \mathrm{L}$ of $50 \mathrm{mM} \mathrm{HCl}$ solution. Then, assay buffer was pumped over the chip for equilibration and the chip was ready for the next run. The transmission spectra of all ten BB-MZIs were recorded sequentially with $1 \mathrm{~s}$ to be the integration time per BB-MZI. The BB-MZI responses were presented as phase shifts in TE polarization after Discrete Fourier Transform of the recorded output spectra. ${ }^{44}$ For the preparation of the calibration curves, the percent ratios of the net calibrator phase shift $\left(S_{x}\right)$ to the net zero calibrator phase shift $\left(\mathrm{S}_{0}\right)$ were plotted against the content of white cheese and cow mozzarella to feta cheese and buffalo mozzarella, respectively.

\section{Results and discussion}

The sensor and the associated immunoassay protocol that were developed for the detection of cheese adulteration with cow milk was employed for testing two types of PDO cheeses, namely mozzarella and feta cheese. Mozzarella is traditionally made of buffalo milk, whereas feta cheese from a mixture of sheep/goat milk (usually 70/30\% w/w). There are several differences in the milk of species from which the two types of cheeses are made as well as the way they are prepared and preserved. Buffalo milk contains more fat than both sheep and goat milk, whereas sheep milk has higher lactose content than buffalo and goat milk. Moreover, feta is preserved in brine with high salt content, while mozzarella is preserved in whey or brine with low salt content. From these differences the two cheeses are expected to constitute two significantly different matrices with different behaviors and effects on the immunoassay and the sensor response. Therefore, the optimization of the assay parameters was performed for each cheese separately.

Prior to the assay optimization, the anti-к-casein rabbit antisera were characterized using an assay for bovine $\kappa$-casein determination previously developed. ${ }^{40}$ At first, the antisera received by the four bleedings were compared in terms of titer by passing over MZIs coated with bovine $\kappa$-casein dilutions of each antiserum ranging from $1: 20$ to $1: 1000$. The antisera titers were determined as the dilutions that provided a phase shift signal of $1 \mathrm{rad}$. It was found that the antiserum received from the 1 st and 2 nd bleeding did not provide a noticeable signal when used at dilutions as low as $1: 50$, whereas the antisera received by the 3 rd and 4 th had a titer of $1: 350$ and $1: 300$, respectively. Thus, the antiserum received from the $3 \mathrm{rd}$ bleeding was employed for the development of assays for bovine cheese detection in feta and buffalo mozzarella.

The specificity of the anti-bovine $\kappa$-casein antiserum with respect to total bovine casein, $\beta$-casein, $\alpha$-casein, $\alpha$-lactalbumin, $\beta$-lactoglobulin, serum albumin and bovine immunoglobulins was also determined. For this purpose, MZIs were coated with bovine $\kappa$-casein and reacted with a
$1: 350$ antiserum dilution in the presence of different concentrations of $\kappa$-casein, whole casein, $\beta$ - and $\alpha$-casein, $\alpha$-lactalbumin, $\beta$-lactoglobulin, serum albumin and bovine immunoglobulins in assay buffer following a previously published assay protocol. ${ }^{40}$ From the inhibition curves obtained (Fig. S2†), percent cross-reactivity values of 13.8 and $6.7 \%$ for whole casein and $\beta$-casein, respectively, were calculated. It should be noted that the cross-reactivity observed for whole casein is expected, since $\kappa$-casein represents $12-15 \%$ of the whole casein. $^{45} \quad$ Regarding $\alpha$-casein, $\alpha$-lactalbumin, $\beta$-lactoglobulin, serum albumin and bovine immunoglobulins, using solutions with a concentration as high as $100 \mu \mathrm{g} \mathrm{mL} \mathrm{m}^{-1}$, there was not any noticeable signal drop, indicating the absence of antibody cross-reactivity against those substances. Thus, the developed antibody is highly specific for bovine к-casein.

\subsection{Optimization of the assay for detection of mozzarella cheese adulteration}

3.1.1. Selection of assay format and sample dilution. A competitive immunoassay format was implemented including the immobilization of bovine $\kappa$-casein onto the sensing arms of the MZIs and then passing a mixture of cheese extract under investigation with the anti-bovine $\kappa$-casein antibody in order to achieve the detection of buffalo mozzarella adulteration with cow milk with the integrated BB-MZI sensor. Therefore, the maximum signal (zero calibrator signal) is observed in the absence of adulteration, whereas a decreased signal is observed in the presence of cow casein in the buffalo mozzarella cheese. Hence, the highest the percent signal drop observed in the case of adulteration, the most sensitive the assay. With respect to the ease of assay implementation in food analysis it would be desirable to detect adulteration during the primary immunoreaction (i.e., the competitive binding reaction of anti-bovine $\kappa$-casein antibody with the $\kappa$-casein fragment, e.g. caseinomacropeptide, in the sample and that immobilized onto the BB-MZIs) for reasons of simplicity and speed; however, the effects of the cheese matrix hindered such an approach. As shown in Fig. 2, it was not possible to distinguish between the response obtained from к-casein coated BB-MZIs from those coated with BSA (blank MZIs) when a zero calibrator of cow cheese in buffalo mozzarella was run at 50-times dilution of the cheese sample, as opposed to the responses obtained for a zero calibrator in buffer. This was mainly due to a significant contribution from the sample matrix, in contrast to the case of the zero calibrator prepared in assay buffer. This effect was observed for all the ranges of sample extract dilutions (1/10-1/200) tested (see ESI Fig. S $3 \dagger$ ) and could be ascribed to the bulk refractive index of the cheese extract as well as to the non-specific binding of several components, such as other proteins or lipids that are inescapably contained at high concentrations in the extract. To overcome this problem, at first different blocking solutions were tested including carbonate or phosphate buffers containing BSA at concentrations ranging from 1 to $3 \%(\mathrm{w} / \mathrm{v})$, diluted goat milk or goat cheese extract. It was found that the 


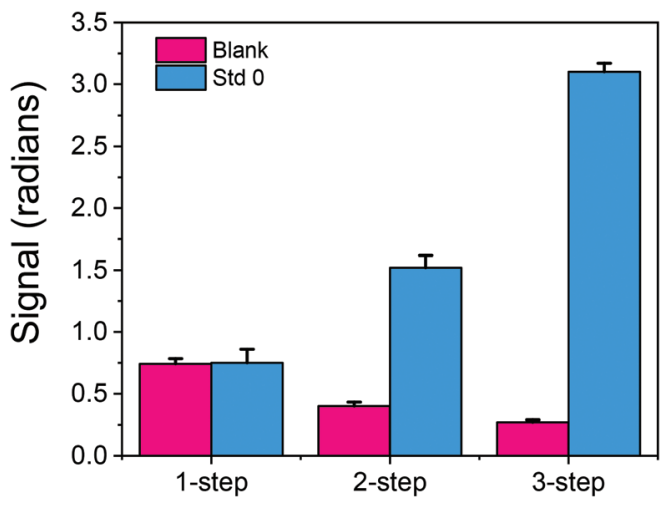

Fig. 2 Responses obtained from bovine $\kappa$-casein coated MZls (light blue bars) or blank MZls (dark pink bars) when running the zero calibrator corresponding to 50-times diluted buffalo mozzarella cheese in a mixture $(1: 1, \mathrm{v} / \mathrm{v})$ with rabbit anti-bovine $\kappa$-casein $\mathrm{Ab}$ (1/100 diluted antiserum) (1-step), followed by a $10 \mu \mathrm{g} \mathrm{mL}^{-1}$ secondary anti-rabbit antibody solution (2-step) or (c) followed by running $10 \mu \mathrm{g} \mathrm{mL}^{-1}$ biotinylated antirabbit antibody and a $10 \mu \mathrm{g} \mathrm{mL}^{-1}$ streptavidin solution (3-step). In all cases the duration of each step was $5 \mathrm{~min}$ and the flow rate was $22 \mu \mathrm{L}$ $\mathrm{min}^{-1}$. Each point is the mean value of 3 measurements \pm SD.

implementation of different blocking solutions did not alter the non-specific signal and, therefore a $1 \%(\mathrm{w} / \mathrm{v})$ BSA solution in $0.1 \mathrm{M} \mathrm{NaHCO}_{3}, \mathrm{pH} 8.5$, was adopted in the final protocol. Then, several assay buffer formulations have been tested to which different surfactants or chaotropic reagents such as Triton-X-100, Tween 20, 0.5 M KCl, EDTA, etc., have been added. Nevertheless, none of the tested buffer formulations was able to suppress the non-specific signal. For this reason, in order to distinguish the response of BB-MZIs coated with bovine $\kappa$-casein from those of blank BB-MZIs and to increase the specific signal, the introduction of further reaction steps was investigated involving the reaction with a secondary antirabbit IgG antibody or a biotinylated secondary antibody in combination with streptavidin. As shown in Fig. 2, both approaches resulted in clear discrimination of the signal received from the $\kappa$-casein coated BB-MZIs from those obtained from the blank sensors. Particularly, the 3-step assay involving a reaction with biotinylated anti-rabbit IgG antibody followed by a reaction with streptavidin resulted in an almost 2-times net signal increase of the zero calibrator and further reduction of the response of the blank MZIs compared to the 2 -step assay. It should be noted that the reaction with streptavidin increased the net signal 2.5 times with respect to the signal received with the secondary antibody. This result was due to multiple labelling of the secondary antibody with biotin moieties allowing binding of several streptavidin molecules per secondary antibody as well as due to the faster kinetics of the streptavidin-biotin reaction compared to the secondaryprimary antibody reaction that counterbalances to some extent the difference of the molecular mass between antibodies $(\sim 150 \mathrm{kDa})$ and streptavidin (55 kDa). In addition, the implementation of biotinylated secondary antibody and streptavidin (3-step assay) did not affect the assay sensitivity com- pared to the assay employing only the reaction with the secondary antibody (2-step assay).

In the first experiments, the duration of each step, i.e. of the primary immunoreaction, the reaction with the secondary antibody (biotinylated or not) and streptavidin, was $5 \mathrm{~min}$. In an attempt to keep the assay duration under 10 min without compromising the absolute signal received with the 3-step assay and due to the fact that the solution was introduced into the sensor using an injector with a $100 \mu \mathrm{L}$ sample loop, the signals received following the 2-step assay for a flow rate of $22 \mu \mathrm{L} \mathrm{min}{ }^{-1}$ (so as each assay step to last $<5 \mathrm{~min}$ ) were compared to those received with the 3-step assay for a flow rate of $33 \mu \mathrm{L} \mathrm{min}{ }^{-1}$ (so as each assay step would last $\sim 3 \mathrm{~min}$ ). As shown in Fig. 3a, even with reduction of both the primary and
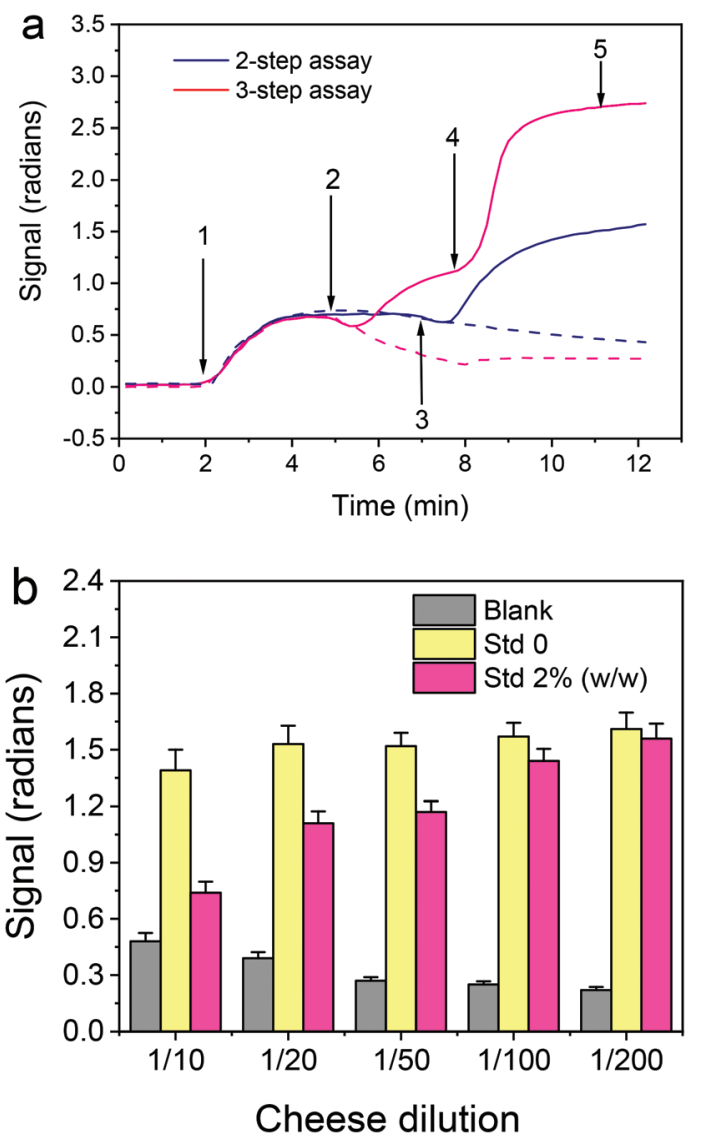

Fig. 3 (a) Responses obtained for the zero calibrator corresponding to 50 -times diluted buffalo mozzarella cheese from bovine $\kappa$-casein coated BB-MZIs (continuous lines) or blank BB-MZIs (dashed lines) following a 2-step (blue lines) or 3-step assay format (magenta line). For the 2-step assay the sequence was: arrow 1-3: a 1:1 mixture of calibrator with 1/100 diluted rabbit antiserum; arrow 3-5: $10 \mu \mathrm{g} \mathrm{mL}^{-1}$ antirabbit antibody solution at a flow rate of $22 \mu \mathrm{L} \mathrm{min}{ }^{-1}$. For the 3-step assay the sequence was: arrow 1-2: a 1:1 mixture of calibrator with 1/ 100 diluted rabbit antiserum; arrow 2-4: $10 \mu \mathrm{g} \mathrm{mL}^{-1}$ anti-rabbit antibody solution; arrow 4-5: $10 \mu \mathrm{g} \mathrm{mL}^{-1}$ streptavidin solution at a flow rate of $33 \mu \mathrm{L} \mathrm{min}{ }^{-1}$. (b) Effect of mozzarella extract dilution with assay buffer on the responses obtained for the zero calibrator (yellow bars) and a calibrator containing $2 \%(\mathrm{w} / \mathrm{w})$ cow mozzarella (magenta bars) as well as from a blank sensor (grey bars) following a 3-step assay format. Each point is the mean value of 3 measurements \pm SD. 
secondary immunoreaction duration from 5 to $3 \mathrm{~min}$, the signal received with the three step assay was $75 \%$ higher than the signal received following the two step assay format.

Once the assay format was selected, the dilution of cheese extract to be used in the assay was determined. As shown in Fig. $3 \mathrm{~b}$, the zero calibrator signal was stable for cheese extract dilution equal to or higher than 20-times, while the blank BB-MZI signal was reduced as the cheese extract dilution increased and acquired minimum values for dilutions equal to or higher than $1 /$ 50. These results in combination with the fact that the $\%$ signal drop obtained for a calibrator containing $2 \%(\mathrm{w} / \mathrm{w})$ bovine mozzarella did not considerably differ for cheese extract dilutions of 1/20 and $1 / 50$, therefore in order to expand the assay dynamic range, $1 /$ 50 dilution was selected for the final assay protocol.

3.1.2. Assay parameter optimization. After the selection of the assay format, additional key parameters were optimized. First, the effect of the flow rate on the signal was investigated using flow rates ranging from 12 to $57 \mu \mathrm{L} \mathrm{min}{ }^{-1}$ (corresponding to the total assay duration ranging from 25 to $5 \mathrm{~min}$, respectively), since a $100 \mu \mathrm{L}$ loop has been used for introduction of each one of the solutions to the chip. As expected, the higher the flow rate, the lower the signal. However, as shown in Fig. 4a, when the flow rate increased from 12 to $33 \mu \mathrm{L} \mathrm{min}{ }^{-1}$
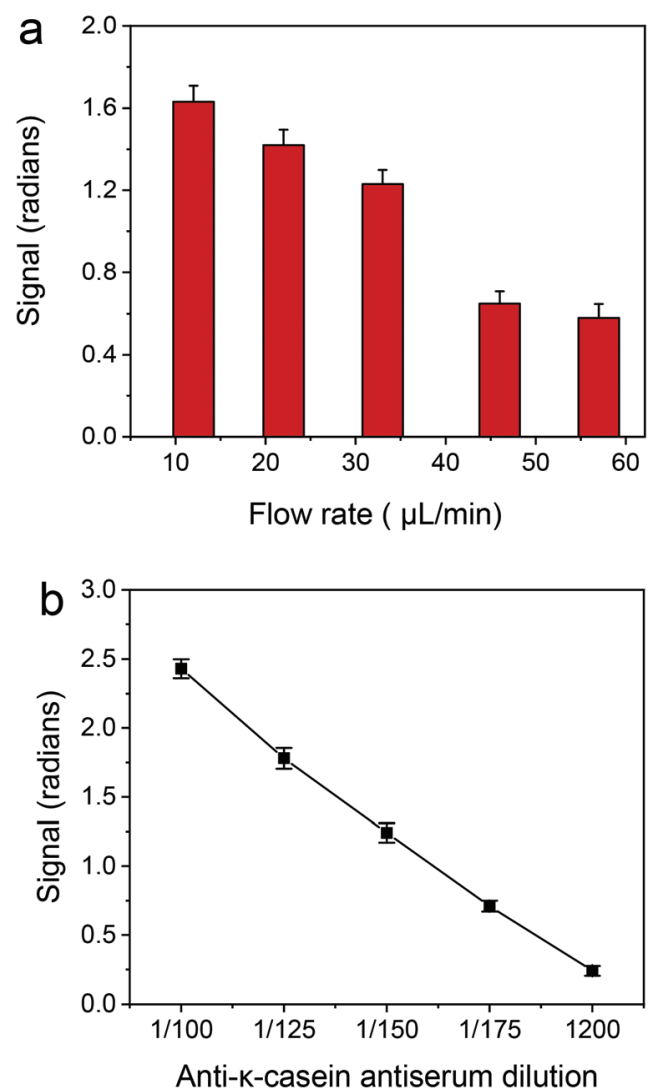

Fig. 4 (a) Effect of flow rate on the net zero calibrator signals using a

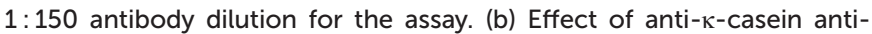
serum dilution on the net zero calibrator signal. The flow rate used throughout the experiment was $33 \mu \mathrm{L} \mathrm{min}{ }^{-1}$. Each point is the mean value of 3 measurements \pm SD. the signal was reduced by approximately $25 \%$, with the assay time being reduced by 2.5 times. On the other hand, a further increase of the flow rate from 33 to $46 \mu \mathrm{L} \mathrm{min}{ }^{-1}$ caused an abrupt signal drop of about $40 \%$ whereas a further flow rate increase of up to $57 \mu \mathrm{L} \mathrm{m^{-1 }}$ caused only a marginal signal drop of less than $10 \%$ compared to that of the $46 \mu \mathrm{L} \mathrm{min}{ }^{-1}$ flow rate. The considerable signal drop observed when the flow rate was increased from 33 to $46 \mu \mathrm{L} \mathrm{min}{ }^{-1}$ could be ascribed not only to the reduction of the reaction time, but also to the fact that the high rate interferes in the binding between the immobilized biomolecules with their counterpart molecules in the liquid running over the chip. Under the light of all the above, the flow rate of $33 \mu \mathrm{L} \mathrm{min}{ }^{-1}$ was selected, since it provided an acceptable compromise between a reasonably short assay duration and a signal range that can offer the desired dynamic range.

In a second step, the optimum antiserum dilution to be used was determined. As shown in Fig. 4b, the zero calibrator signal dropped as the antiserum dilution used in the assay increased from $1: 100$ to $1: 200$ (corresponding to dilution of $1: 200-1: 400$ in the reaction mixture, respectively). At the same time the assay sensitivity, i.e., the percent signal drop obtained for calibrators containing cow cheese increased, as the antiserum dilution was increased from $1: 100$ to $1: 200$. Therefore, as a trade-off between the absolute signal and the assay sensitivity a 1:150 antiserum dilution was selected for the final protocol.

Finally, another aspect investigated was the potential of chip regeneration after the assay completion, i.e., the removal of the bound to immobilized $\kappa$-casein molecules (primary antibody, secondary antibody, and streptavidin), so as to render the chip ready for a new assay cycle, an attribute that can considerably reduce the cost of the chip as a consumable. Based on the previous experience with regard to regenerating and re-using the BB-MZI chips, ${ }^{34-37}$ a $50 \mathrm{mM} \mathrm{HCl}$ and a $40 \mathrm{mM}$ $\mathrm{NaOH}$ solution were tested. It was found that neither of them resulted in adequate removal of the bound biomolecules. However, as shown in the real-time response graph presented in Fig. S4, $\uparrow$ complete removal of the bound biomolecules was achieved by passing at first a $40 \mathrm{mM}$ $\mathrm{NaOH}$ solution (which removed almost $80 \%$ of the bound molecules) followed by a $50 \mathrm{mM} \mathrm{HCl}$ solution. Therefore, for the detection of adulteration of buffalo mozzarella with cow milk, regeneration can be achieved by running sequentially over the chip $40 \mathrm{mM} \mathrm{NaOH}$ and $50 \mathrm{mM} \mathrm{HCl}$ for $3 \mathrm{~min}$ each. Applying the selected regeneration procedure, repetitive assay/regeneration cycles have been performed in the $\kappa$-casein coated chips. In Fig. 5, the real-time response during two assay cycles, one prior to and one after regeneration, is presented. As shown, the response obtained prior to regeneration is the same as that obtained after regeneration. Moreover, as shown in Fig. S5, $\uparrow$ where the net zero calibrator values obtained from a single chip for 11 repetitive assay/ regeneration cycles are provided, the values for the first 9 assay cycles were within the mean value \pm 2 SD limits, indicating that each chip could be used up to 9 times. 


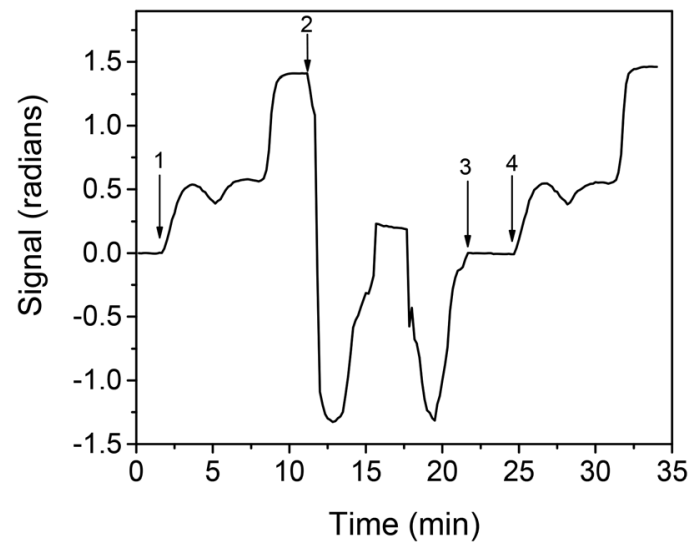

Fig. 5 Real-time response obtained for the zero calibrator for two repetitive assay cycles, one prior to regeneration (arrows 1-2) and another after regeneration (arrow 4 to end). Regeneration (arrows 2-3) was followed by equilibration with assay buffer (arrows 3-4) prior to start of a new assay cycle.

Once the assay parameters were set, calibrators containing 0.5 to $50 \%$ cow cheese in buffalo mozzarella were prepared and run over the chip. The real-time responses provided by the bovine $\kappa$-casein coated MZIs are depicted in Fig. S6† along with the response obtained from a blank sensor, whereas in Fig. 6a the net responses obtained after subtraction of the blank sensor response are presented. As shown, although the differences in the signal obtained for the different calibrators were not easily discernible during the primary immunoreaction they were easily distinguished both during the subsequent reaction with the biotinylated secondary antibody and even more during the reaction with streptavidin. The calibration curve of the assay is depicted in Fig. $6 \mathrm{~b}$.

3.1.3. Analytical characteristics of the assay for mozzarella adulteration detection. From the linearized calibration curve presented in Fig. 6b, the assay detection and quantification limits were determined as the cow cheese percentage corresponding to the signal equal to 100-3SD and 100-6SD of the mean zero calibrator signal obtained from the measurements of 21 MZIs and were 0.25 and $0.5 \%$, respectively. The intraand inter-assay coefficients of variation (CV) have been determined by preparing three samples containing $0.7,7.0$ and $20 \%(\mathrm{w} / \mathrm{w})$ cow cheese. In particular, the intra-assay CV was determined by running each sample 3 times in a single day (21 sensors in total), while the inter-assay CV was calculated by running each sample in duplicate in five different days over a period of one month, and found to be less than 7.6 and $8.9 \%$, respectively.

\subsection{Optimization of feta cheese adulteration detection}

The optimization of the assay for detection of feta cheese adulteration with cow milk included the optimization of the same parameters as for buffalo mozzarella adulteration detection. It was found that the optimum conditions with respect to rabbit anti-bovine $\kappa$-casein dilution, the flow rate of the reagents, the cheese extract dilution, and the assay format and duration
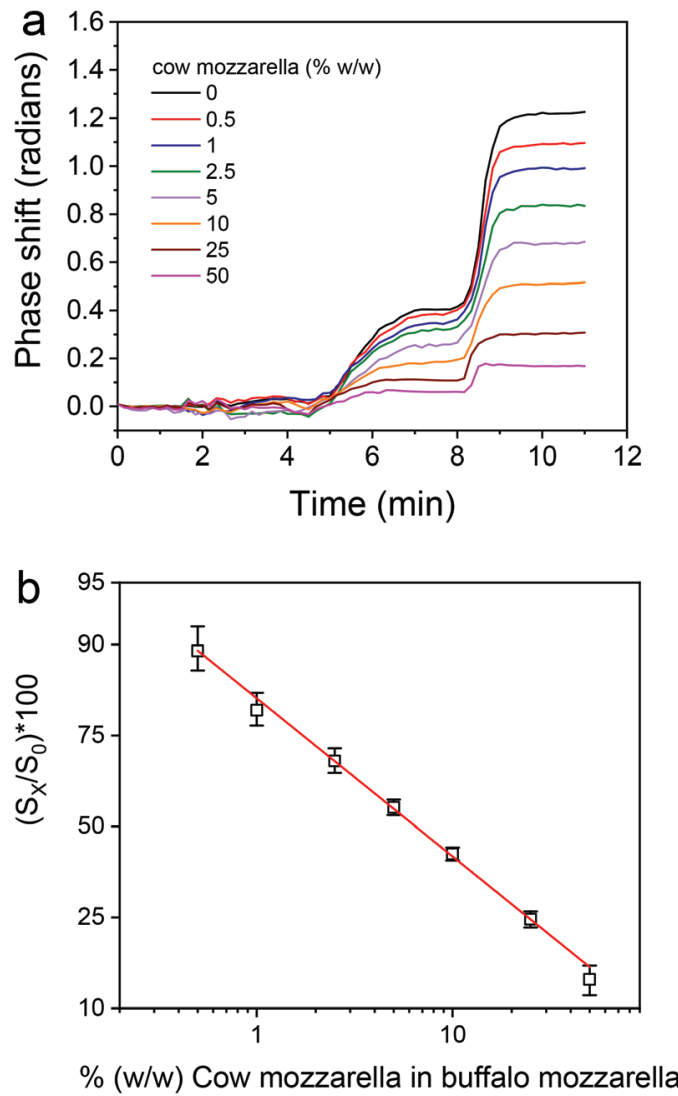

Fig. 6 (a) Net real-time signal responses obtained for cow cheese calibrators $(0-50 \%, w / w)$ in the mozzarella cheese matrix. (b) Typical calibration curve obtained for mozzarella cheese adulteration with cow cheese. Each point is the mean value of 3 measurements \pm SD.

were the same as those determined for mozzarella. Nonetheless, there was a clear difference in the matrix effect between feta and mozzarella, since as shown in Fig. S7, $\dagger$ where the real-time responses from bovine $\kappa$-casein coated BB-MZIs corresponding to different calibrators are provided along with the response of a blank sensor, the blank sensor response returned to values close to zero after the primary immunoreaction, i.e., during the reaction with the biotinylated secondary antibody and streptavidin. Regardless of that, as in the case of mozzarella, it was not possible to distinguish the signal corresponding to different calibrators by monitoring the primary immunoreactions, even after subtraction of the blank sensor response (Fig. 7a), whereas it was easy to distinguish between the different calibrators during the reaction with the biotinylated secondary antibody and the streptavidin. A typical linearized calibration curve is provided in Fig. 7b, from which it is deduced that the linear response range of the assay extents from 0.25 to $25 \%$ cow milk in feta cheese.

From the linearized calibration curve presented in Fig. 7b, the assay detection and quantification limits were determined as the cow cheese percentage corresponding to the signal equal to 100-3SD and 100-6SD of the mean zero calibrator signal obtained from the measurements of 21 MZIs and were 0.125 and $0.25 \%(\mathrm{w} / \mathrm{w})$, respectively. 

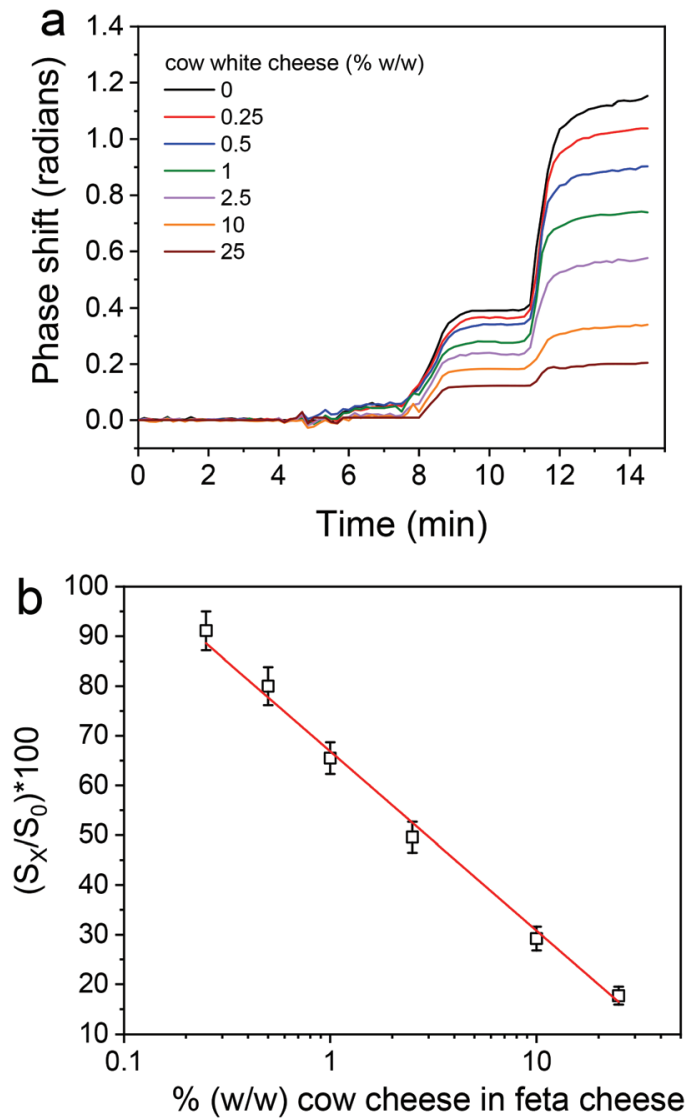

Fig. 7 (a) Net real-time signal responses obtained for cow cheese calibrators $(0-25 \%, w / w)$ in the feta cheese matrix. (b) Typical calibration curve obtained for feta cheese adulteration with cow cheese. Each point is the mean value of 3 measurements $\pm S D$.

The intra- and inter-assay coefficients of variation (CV) have been determined by preparing three samples containing 0.7 , 7.0 and $20 \%(\mathrm{w} / \mathrm{w})$ cow cheese. In particular, the intra-assay CV was determined by running each sample 3 times in a single day (21 sensors in total), while the inter-assay CV was calculated by running twice each sample in five different days, and they were found to be less than 6.8 and $9.2 \%$, respectively.

\section{Conclusions}

A rapid and sensitive silicon photonic immunosensor for the detection of buffalo mozzarella and feta cheese adulteration with bovine milk has been developed using an in-house antibovine $\kappa$-casein rabbit antiserum. The quantification limits determined for bovine cheese detection in mozzarella and feta were 0.5 and $0.25 \%(\mathrm{w} / \mathrm{w})$, respectively, allowing adulteration detection at levels below the allowable $1 \%(\mathrm{w} / \mathrm{w})$ limit for bovine milk presence in cheeses prepared from the milk of species other than cows as set by the EU regulations. In addition to the increased sensitivity, the sensor has a broad linear dynamic range, up to $50 \%(\mathrm{w} / \mathrm{w})$ for mozzarella and $25 \%$ (w/w) for feta, allowing detection along this range with a fixed dilution of cheese extract. The sensor is, to the best of our knowledge, the first sensor reported so far in the literature that addresses this subject of significant economic and health implications, and it is also the first that combines short testing times with a low detection limit. The fact that the silicon photonic sensor can be applied to detect adulteration with bovine milk in two cheeses that are very different regarding their composition as well as the method of their preparation and preservation is indicative of its potential for adulteration detection in other kinds of cheese and widespread application in dairy product quality controls.

\section{Conflicts of interest}

There are no conflicts to declare.

\section{Acknowledgements}

M. Angelopoulou was supported by the program of Industrial Scholarships of Stavros Niarchos Foundation. The authors wish to thank the personnel of the Animal House of NCSR "Demokritos" for excellent technical assistance and the technical staff of the Nanotechnology and Microsystems Laboratory of INN, part of the "National Infrastructure in Nanotechnology, Advanced Materials and Micro-/ Nanoelectronics" INNOVATION-EL (MIS 5002772) which is implemented under the Action "Reinforcement of the Research and Innovation Infrastructure", funded by the Operational Programme "Competitiveness, Entrepreneurship and Innovation" (NSRF 2014-2020) and co-financed by Greece and the European Union (European Regional Development Fund). This work was also supported in part by the EU-funded Project "FOODSNIFFER” (FP7-ICT-318319).

\section{References}

1 K. Raynal-Ljutovac, G. Lagriffoul, P. Paccard, I. Guillet and Y. Chilliard, Small Rumin. Res., 2008, 79, 57-72.

2 H. K. Mayer, Int. Dairy J., 2005, 15, 595-604.

3 M. Borkova and J. Snaselova, Czech J. Food Sci., 2005, 23, 41-50.

4 A. K. Seçkin, B. Yilmaz and H. Tosun, LWT-Food Sci. Technol., 2017, 77, 332-336.

5 R. E. Goodman, S. L. Taylor, J. Yamamura, T. Kobayashi, H. Kawakami, C. L. Kruger and G. P. Thompson, Food Chem. Toxicol., 2007, 45, 1787-1794.

6 B. Shatenstein and P. Ghadirian, Nutrition, 1998, 14, 223230.

7 European Parliament and Council, Off. J. Eur. Commun., 2000, L109, 29-42.

8 European Parliament and Council, Off. J. Eur. Commun., 2003, L308, 15-18.

9 Food Allergen Labeling and Consumer Protection Act of 2004, Public Law 108-282, August 2, 2004. 
10 Commission Regulation EC No 273/2008 of 5 March 2008, Off. J. Eur. Commun., 2008, L88, 53-61.

11 N. A. Chávez, E. Salinas, J. Jauregui, L. A. Palomares and K. Macías, Food Agric. Immunol., 2008, 19, 265-272.

12 B. H. R. F. Gonçalves, G. de J. Silva, S. F. O. Pontes, R. da C. I. Fontan, A. S. Egito and S. P. B. Ferrão, Int. J. Food Sci. Technol., 2016, 51, 1586-1593.

13 G. Enne, D. Elez, F. Fondrini, I. Bonizzi, M. Feligini and R. Aleandri, J. Chromatogr. A, 2005, 1094, 169-174.

14 S. Camerini, E. Montepeloso, M. Casella, M. Crescenzi, R. M. Marianella and F. Fuselli, Food Chem., 2016, 197, 1240-1248.

15 C. Dal Bosco, S. Panero, M. A. Navarra, P. Tomai, R. Curini and A. Gentili, J. Agric. Food Chem., 2018, 66, 5410-5417.

16 T. M. C. Motta, R. B. Hoff, F. Barreto, R. B. S. Andrade, D. M. Lorenzini, L. Z. Meneghini and T. M. Pizzolato, Talanta, 2014, 120, 498-505.

17 R. Cozzolino, S. Passalacqua, S. Salemi and D. Garozzo, J. Mass Spectrom., 2002, 37, 985-991.

18 C. D. Calvano, C. De Ceglie, A. Monopoli and C. G. Zambonin, J. Mass Spectrom., 2012, 47, 1141-1149.

19 C. Agrimonti, A. Pirondini, M. Marmiroli and N. Marmiroli, Food Chem., 2015, 187, 58-64.

20 M. Feligini, I. Bonizzi, V. C. Curik, P. Parma, G. F. Greppi and G. Enne, Food Technol. Biotechnol., 2005, 43, 91-95.

21 I. Ganopoulos, I. Sakaridis, A. Argiriou, P. Madesis and A. Tsaftaris, Food Chem., 2013, 141, 835-840.

22 I. P. Hurley, R. C. Coleman, H. E. Ireland and J. H. H. Williams, Int. Dairy J., 2006, 16, 805-812.

23 I. M. López-Calleja, I. González, V. Fajardo, P. E. Hernández, T. García and R. Martín, Int. Dairy J., 2007, 17, 87-93.

24 N. Stănciuc and G. Râpeanu, Food Agric. Immunol., 2010, 21, 157-164.

25 H. Colak, A. Aydin, B. Nazli and O. Ergun, Food Control, 2006, 17, 905-908.

26 P. Galan-Malo, I. Mendiara, P. Razquinand and L. Mata, Food Addit. Contam., Part A, 2018, 35, 599-604.

27 C. Crosson and C. Rossi, Biosens. Bioelectron., 2013, 42, 453-459.

28 S. Eissa, C. Tlili, L. L'Hocine and M. Zourob, Biosens. Bioelectron., 2012, 38, 308-313.

29 Q. Cao, H. Zhao, Y. Yang, Y. He, N. Ding, J. Wang, Z. Wu, K. Xiang and G. Wang, Biosens. Bioelectron., 2011, 26, 34693474 .
30 S. Muller-Renaud, D. Dupont and P. Dulieu, Food Agric. Immunol., 2003, 15, 265-277.

31 W. Haasnoot, G. R. Marchesini and K. Koopal, J. AOAC Int., 2006, 89, 849-855.

32 R. Pilolli, L. Monaci and A. Visconti, Trends Anal. Chem., 2013, 47, 12-26.

33 P. Kozma, A. Hamori, S. Kurunczi, K. Cottier and R. Horvath, Sens. Actuators, B, 2011, 155, 446-450.

34 D. Patko, K. Cottier, A. Hamori and R. Horvath, Opt. Express, 2012, 20, 23162.

35 A. B. Gonzalez-Guerrero, J. Maldonado, S. Herranz and L. M. Lechuga, Anal. Methods, 2016, 8, 8380-8394.

36 W. Haasnoot, N. G. E. Smits, A. E. M. Kemmers-Voncken and M. G. E. G. Bremer, J. Dairy Res., 2004, 71, 322-329.

37 M. Angelopoulou, A. Botsialas, A. Salapatas, P. S. Petrou, W. Haasnoot, E. Makarona, J. Gerhard, D. Goustouridis, A. Siafaka-Kapadai, I. Raptis, K. Misiakos and S. E. Kakabakos, Anal. Bioanal. Chem., 2015, 407, 39954004.

38 A. Psarouli, A. Botsialas, A. Salapatas, G. Stefanitsis, D. Nikita, G. Jobst, N. Chaniotakis, D. Goustouridis, E. Makarona, P. S. Petrou, I. Raptis, K. Misiakos and S. E. Kakabakos, Talanta, 2017, 165, 458-465.

39 V. Pagkali, P. S. Petrou, E. Makarona, J. Peters, W. Haasnoot, G. Jobst, I. Moser, K. Gajos, A. Budkowski, A. Economou, K. Misiakos, I. Raptis and S. E. Kakabakos, J. Hazard. Mater., 2018, 359, 445-453.

40 M. Angelopoulou, P. S. Petrou, E. Makarona, W. Haasnoot, I. Moser, G. Jobst, D. Goustouridis, M. Lees, K. Kalatzi, I. Raptis, K. Misiakos and S. E. Kakabakos, Anal. Chem., 2018, 90, 9559-9567.

41 N. A. Chávez, J. Jauregui, L. A. Palomares, K. E. Macías, M. Jiménez and E. Salinas, Dairy Sci. Technol., 2012, 92, 121-132.

42 D. Tsounidi, G. Koukouvinos, P. Petrou, K. Misiakos, G. Zisis, D. Goustouridis, I. Raptis and S. E. Kakabakos, Sens. Actuators, B, 2019, 282, 104-111.

43 E. Livaniou, G. P. Evangelatos and D. S. Ithakissios, Clin. Chem., 1987, 33, 1983-1988.

44 K. Misiakos, I. Raptis, A. Salapatas, E. Makarona, A. Botsialas, M. Hoekman, R. Stoffer and G. Jobst, Opt. Express, 2014, 22, 8856-8870.

45 D. T. Davies and J. R. Law, J. Dairy Res., 1977, 44, 447454. 